\title{
Viel Luft nach oben
}

\section{Große Engagement-Potenziale in der Freien Wohlfahrtspflege}

\author{
HOLGER BACKHAUS-MAUL, \\ PETER FRIEDRICH, \\ MAUD KROHNIST, \\ MIRIAM HÖRNLEIN UND \\ KARSTEN SPECK \\ Holger Backhaus Holger ist Soziolo- \\ ge und Verwaltungswissenschaftler \\ an der Martin-Luther-Universität \\ Halle-Wittenberg. Peter Friedrich \\ ist Erziehungswissenschaftler an \\ der Martin-Luther-Universität \\ Halle-Wittenberg. Maud Krohn ist \\ Erziehungswissenschaftlerin an \\ der Carl von Ossietzky Universität \\ Oldenburg. Miriam Hörnlein ist \\ Erziehungswissenschaftlerin an der \\ Martin-Luther-Universität Halle- \\ Wittenberg. Prof. Dr. Karsten Speck \\ ist Erziehungswissenschaftler an \\ der Carl von Ossietzky Universität \\ Oldenburg. \\ www.philfakz.uni-halle.de/ \\ paedagogik/rvo
}

\author{
Das Reservoir für freiwilliges Engagement scheint in den \\ Organisationen der Freien Wohlfahrtspflege noch lange \\ nicht ausgeschöpft. Dies ist eines der zentralen Ergebnisse \\ einer neuen empirischen Untersuchung zum ehrenamtlichen \\ Einsatz im Paritätischen Wohlfahrtsverband.
}

Die folgenden Ausführungen eröffnen einen ersten Einblick in eine umfangreiche empirische Untersuchung der Engagement-Potenziale in der Freien Wohlfahrtspflege am Beispiel des Paritätischen Wohlfahrtsverbandes. Die Untersuchung wurde in den Jahren 2012 bis 2014 durchgeführt und soeben abgeschlossen. (1) Forschungsleitend waren dabei die folgenden Fragestellungen:

- Welche Vorstellungen und Deutungen von Engagement und welche Ideen und Perspektiven zur Entwicklung von Engagement existieren in den Mitgliedsorganisationen der Freien Wohlfahrtspflege?

- Welche Bedeutung, welchen Umfang und welche Ausprägungen hat Engagement in den Mitgliedsorganisationen der Freien Wohlfahrtspflege?

- Wie wird Engagement-Förderung in den Mitgliedsorganisationen der Freien Wohlfahrtspflege gesteuert und koordiniert und welche Rahmenbedingungen zur EngagementFörderung werden bereitgestellt?

Um den Paritätischen Wohlfahrtsverband angesichts seiner Größe und Vielfalt untersuchen zu können, wurden drei maximal kontrastierende Landesverbände - Berlin, Nordrhein-Westfalen und Thüringen - ausgewählt. Diese Landesverbände mit ihren Mitgliedsorganisationen bilden in ihrer Differenziertheit den Paritätischen Wohlfahrtverband in seiner heterogenen Gesamtheit treffend ab und tragen zugleich ländlichen als auch klein-, mittel- und großstädtischen Verdichtungsräumen bei einer insgesamt re- lativ günstigen räumlich-geographischen Verteilung im Bundesgebiet Rechnung.

Die Untersuchung basiert auf einer Kombination von quantitativen und qualitativen Forschungsmethoden: In den Mitgliedsorganisationen der Landesverbände des Paritätischen Wohlfahrtsverbandes wurden mittels OnlineBefragung eine grundlegende Bestandserhebung des Engagements durchgeführt und im Rahmen qualitativer Experteninterviews und Gruppendiskussionen wurden die engagementbezogenen Vorstellungswelten und Handlungspraktiken eingehend untersucht.

Zuerst wurden Experteninterviews und Gruppendiskussionen mit 66 Führungs- und Leitungskräften aus Mitgliedsorganisationen unterschiedlicher Arbeitsbereiche sowie mit 17 verbandsinternen und 5 verbandsexternen Experten durchgeführt. Im Mittelpunkt der Experteninterviews und Gruppendiskussionen standen $u$. a. die engagementbezogenen Erfahrungen und Vorstellungen sowie Erwartungen, Vorhaben und Handlungspraktiken der Befragten.

Um in den Experteninterviews die Vielfalt der Mitgliedsorganisationen des Paritätischen Wohlfahrtsverbandes erfassen zu können, wurden vorab Auswahlkriterien für die zu untersuchenden Organisationen festgelegt. Arbeitsbereiche der Freien Wohlfahrtspflege, Rechtsform, Wirkungsumfeld, Personalstruktur und Anzahl der hauptamtlichen Mitarbeiter spielten hierbei eine Rolle.

Die darauf aufbauende OnlineBefragung in den drei Landesverbänden Berlin, Nordrhein-Westfalen und Thüringen richtete sich an insgesamt 
3.957 Organisationen, von denen 2.276 Organisationen $(58 \%)$ an der Befragung teilgenommen haben. Im Mittelpunkt der Online-Befragung standen die Bedeutung, der Umfang und die spezifischen Ausprägungen sowie die organisatorischen Rahmenbedingungen des Engagements in den Verbänden und ihren Mitgliedsorganisationen.

Im Folgenden werden - in Kenntnis der qualitativen Befunde - ausgewählte quantitative Befunde aufgezeigt, die für die ausstehende Diskussion über die Engagement-Entwicklung in der Freien Wohlfahrtspflege insgesamt von Bedeutung sind.

\section{Bedeutung des Engagements}

In den Organisationen der drei untersuchten Landesverbände sind Engagierte eine wichtige und kaum zu überschätzende Größe. Ohne sie könnte ein Großteil der Organisationen seine Aufgaben nicht wahrnehmen oder hätte als Organisation gar keinen Bestand. Über die Hälfte der befragten Organisationen kann ihre Aufgaben nur mit Hilfe von Engagierten realisieren und ein Fünftel der befragten Organisationen ist zumindest in Teilen von den Leistungen Engagierter abhängig (vgl. Abb. 1).

Die Mitgliedsorganisationen in den drei Landesverbänden haben insgesamt über 89.000 Engagierte. Für drei Viertel dieser Engagierten machen die Befragten genaue Angaben zu den Leistungen, die sie mit über 560.000 Stunden im Monat veranschlagen. (2) Neben der inhaltlichen und finanziellen Bedeutung des Engagements schätzt ein Großteil der Organisationen besonders die hohe Motivation und die Kenntnisse der Engagierten. Weitere Aspekte, die genannt werden, sind die Möglichkeiten

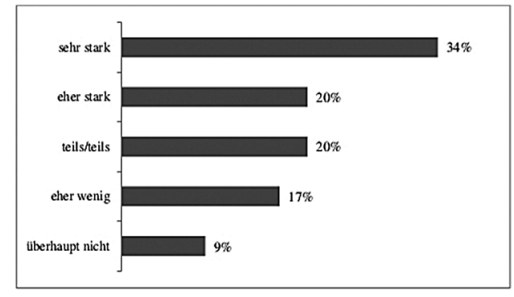

Abb. 1: Für viele der befragten soziale Organisationen stellt die Leistungen der Engagierten eine tragende Größe dar.

$\mathrm{n}=1.713$ (Backhaus-Maul/Speck/Hörnlein/Krohn 2014)

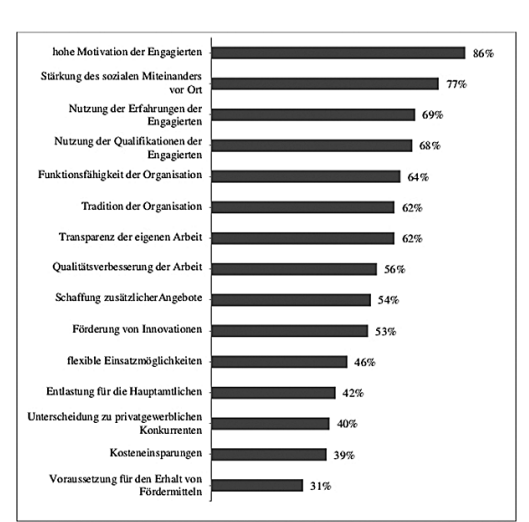

Abb. 2. Besonders die hohe Motivation und die Kenntnisse der Engagierten werden in den befragten sozialen Organisationen geschätzt.

n $=1.375-1.659$, Skala $1-5$ (1 = »überhaupt nicht wichtig", 2 = "eher nicht wichtig", 3 = "teils/teils", 4 = "eher wichtig", 5 = "sehr wichtig"): hier Position 4 und 5 dargestellt (BackhausMaul/Speck/Hörnlein/Krohn 2014)

der Ressourceneinsparung und förderpolitischen Legitimation (vgl. Abb. 2).

\section{Arten und Tätigkeitsfelder des Engagements}

Die Mehrheit aller befragen Organisationen bindet Engagierte sowohl in Gremien als auch im operativen Bereich. Auffällig ist, dass in etwa einem Drittel der Organisationen Engagierte ausschließlich in Gremien tätig sind. Nur $4 \%$ der befragten Organisationen arbeiten ausschließlich mit operativ tätigen Engagierten.

Dieser Befund spiegelt sich auch in den Tätigkeitsfeldern der Engagierten wieder. So befassen sich Engagierte vor allem mit der Organisation und Durchführung von Veranstaltungen sowie praktischen Arbeiten gefolgt von Gremien- und Leitungsarbeit, Öffentlichkeitsarbeit und Verwaltungstätigkeiten (vgl. Abb. 3).

\section{Alters-, Geschlechter- und Zeitstruktur des Engagements}

Hinsichtlich der Altersstruktur wird deutlich, dass über die Hälfte der Engagierten zwischen 31 und 65 Jahre alt ist. Ein Viertel aller angegebenen Engagierten ist älter als 65 Jahre. $15 \%$ der Engagierten sind 30 Jahre alt oder jünger. Damit sind die Engagierten in den drei untersuchten Landesverbänden des Paritätischen Wohlfahrtsverbandes - im Vergleich mit anderen Untersuchungen in der verbandlichen Wohlfahrtspflege - relativ jung. (3)

In der geschlechtsspezifischen Verteilung der Engagierten wird deutlich, dass Frauen mit zwei Dritteln die Mehrheit der Engagierten darstellen (vgl. Abb. 4). Im Vergleich zum Caritas und Diakonie, wo Frauen etwa drei Viertel der Engagierten ausmachen, sind im Paritätischen Wohlfahrtsverband mehr Männer engagiert. (4)

\section{Klassische und "neue" Engagement-Formen}

Den Angaben der befragten Organisationen zufolge erbringen rund 66.600 Engagierte (5) Leistungen im Umfang von über 560.000 Stunden im Monat, wobei die Zahl der Stunden, die die Engagierten in den Organisationen leisten, stark variiert. Knapp zwei Drittel der Engagierten sind kontinuierlich tätig. Lediglich ein reichliches Drittel arbeitet gelegentlich oder punktuell in den befragten Organisationen mit. Somit wird deutlich, dass das klassische kontinuierliche Engagement weiterhin die vorherrschende Engagement-Form darstellt, während neue - befristete - EngagementFormen in einigen Mitgliedsorganisationen im Mittelpunkt stehen.

Die differenzierte Analyse zeigt: Es existiert ein schwacher positiver Zusammenhang zwischen dem Organisationsanteil der Engagierten im Alter ab 46 Jahren und dem Organisationsanteil an kontinuierlichem Engagement. Je mehr

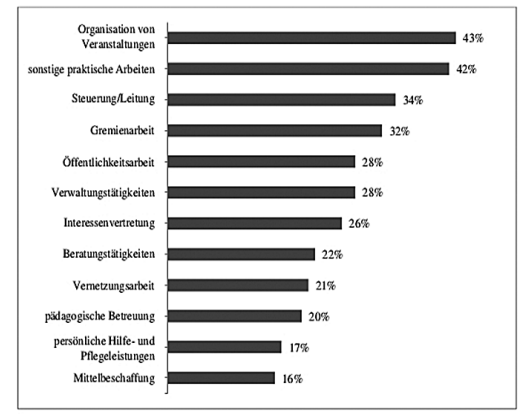

Abb. 3: Die Mehrheit aller befragen Organisationen bindet Engagierte sowohl in Gremien als auch im operativen Bereich.

$\mathrm{n}=1.676-1.719$, Skala $1-5$ (1 = »überhaupt nicht", 2 = "eher wenig", 3 = "teils/teils", 4 = "eher stark", 5 = "sehr stark "): hier Position 4 und 5 dargestellt (Backhaus-Maul/Speck/Hörnlein/ Krohn 2014) 


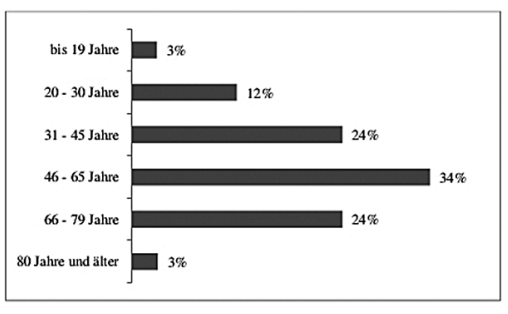

Abb. 4: In den befragten sozialen Organisationen ist über die Hälfte der Engagierten zwischen 31 und 65 Jahre alt ist. $\mathrm{n}=1.728$ (Backhaus-Maul/Speck/Hörnlein/Krohn 2014)

Engagierte in der Altersgruppe ab 46 Jahre die Organisationen aufweisen, desto ausgeprägter ist das kontinuierliche Engagement in der Organisation.

Je mehr Engagierte die Organisation hingegen im Alter zwischen 20 und 45 Jahren einbinden, desto mehr gelegentliches beziehungsweise befristetes Engagement findet sich in den Organisationen. Der Befund eines Zusammenhangs zwischen dem Alter der Engagierten und einem punktuellen Einsatz in den Organisationen scheint den proklamierten sozialen Wandel des Engagements und der Engagement-Formen wiederzugeben. Zugleich ist zu betonen, dass dieser Zusammenhang schwach ausgeprägt ist und somit tiefergehende qualitative Untersuchungen notwendig wären, um einen Trend zum "neuen « befristeten Engagements bei »jüngeren« Engagierten ausreichend zu belegen.

\section{Steuerung und Koordination von Engagement}

Eine gezielte Steuerung und Koordination von Engagement, die mit entsprechenden Instrumenten und Verfahren sowie konkreten Vorhaben unterlegt ist, erweist sich in den befragten Organisationen in der Regel als eher wenig ausgeprägt (vgl. Abb. 5).

In etwa zwei Dritteln aller Organisationen gibt es zwar »EngagementVerantwortliche " und damit Ansätze einer formalen Struktur der Engagement-Förderung. Es zeigt sich aber, dass viele Organisationen, die EngagementVerantwortliche mit geringer Arbeitszeit und Ressourcenausstattung einsetzen sowie über keine besonders ausgeprägte Steuerung und Koordination des Engagements verfügen. Die Benennung einer personellen Zuständigkeit erfordert damit einhergehend eine hinreichende Ausstattung dieser Stellen mit Entscheidungs- und Fachkompetenzen sowie Geld- und Zeitressourcen.

\section{Gewinnung von Engagierten}

Rund die Hälfte der Organisationen ist mit ihrer eigenen Engagement-Förderung zufrieden. Als Gründe für eine »leichte" Unzufriedenheit mit der EngagementFörderung werden fehlende Leitlinien und Ziele für das Engagement sowie eine mangelnde Steuerung und Koordination desselben genannt, nicht aber ein Mangel an Ressourcen. Weiterhin fällt auf, dass Organisationen, die Engagierte ausschließlich in Gremienebenen binden, bisher allenfalls über eine gering ausgeprägte Engagement-Förderung verfügen.

Eine besondere Aufgabe stellt bei der Hälfte der befragten Organisationen die Gewinnung von Engagierten, besonders von jungen Engagierten sowie von Engagierten für Leitungsfunktionen dar. Organisationen im ländlich-kleinstädtischen Umfeld weisen dabei auf stärkere Probleme bei der Gewinnung von Engagierten hin, während großstädtische Organisationen eher seltener die Gewinnung von neuen Engagierten problematisieren (vgl. Abb. 6).

Für die Gewinnung von Engagierten gibt es verschiedene Möglichkeiten - von persönlicher Ansprache bis hin zur $\mathrm{Zu}$ -

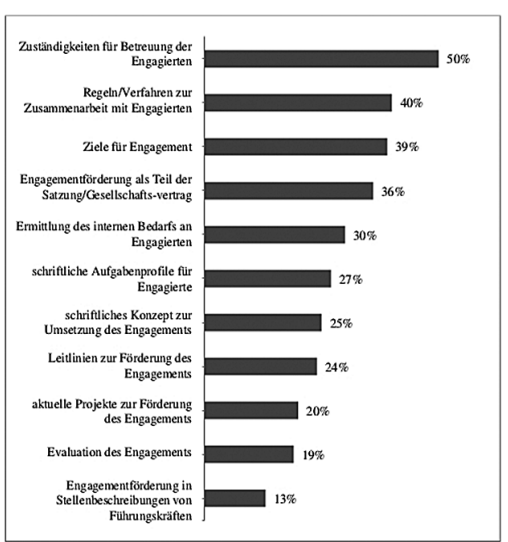

Abb. 5: Eine gezielte Steuerung und Koordination von Engagement ist in den meisten befragten sozialen Organisationen kaum ausgeprägt.

$n=1.609-1.635$, Skala $1-5(1=$ "trifft überhaupt nicht zu", 2 = "trifft eher nicht zu", 3 = "teils/teils", 4 = "trifft eher zu«, 5 = "trifft völlig zu«): hier Position 4 und 5 dargestellt (BackhausMaul/Speck/Hörnlein/Krohn 2014)

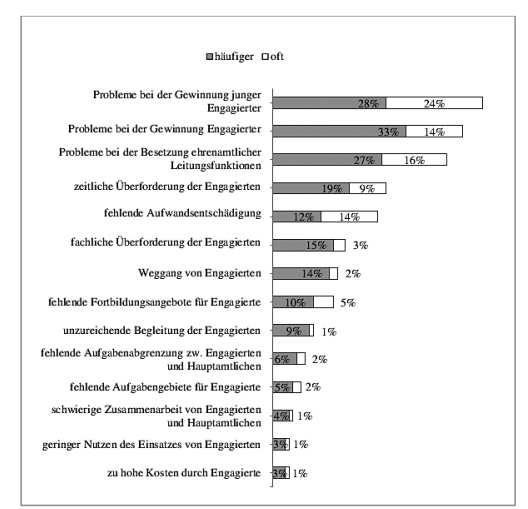

Abb. 6: Rund die Hälfte der befragten sozialen Organisationen ist mit ihrer eigenen Engagement-Förderung zufrieden. $\mathrm{n}$ (gesamt) $=1.375-1.681, \mathrm{n}$ (mit Hauptamtlichen $)=1.375-1.382$, Skala $1-5(1$ = "überhaupt nicht", 2 = "selten", 3 = "manchmal", 4 = »häufiger", 5 = "oft"): hier Position 4 und 5 dargestellt (Backhaus-Maul/Speck/Hörnlein/Krohn 2014)

sammenarbeit mit Vermittlungs- und Kontaktstellen für Engagement. Die Mehrheit der befragten Organisationen greift am ehesten auf Möglichkeiten der persönlichen Ansprache zurück. Nur wenige der befragten Organisationen nutzen interaktiv-kooperative Ansätze, wie die Zusammenarbeit mit Vermittlungs- und Kontaktstellen für Engagement, eigene Beratungsangebote für Interessenten oder eine gezielte Öffentlichkeitsarbeit; die Mehrzahl der Organisationen ist demgegenüber nicht oder kaum in die lokale Bürgergesellschaft und EngagementInfrastruktur eingebunden (vgl. Abb. 7).

\section{Unausgeschöpfte Engagement-Potenziale}

Wenn sich der skizzierte Befund zur Gewinnung von Engagierten erhärtet, nachdem die Organisationen der Freien Wohlfahrtspflege zu großen Teilen kein integraler Bestandteil der lokalen Bürgergesellschaft und EngagementInfrastruktur sind, so deutet sich hier - neben einer sachlichen und legitimatorischen Lücke - ein erhebliches unausgeschöpftes Engagement-Potenzial in ihrer unmittelbaren sozialen Umwelt an. Aber selbst innerhalb der befragten Organisationen besteht ein markanter »blinder Fleck« in punkto EngagementPotenzial, um das Parteien und Verbände den Paritätischen Wohlfahrtsverband geradezu »beneiden « würden, wenn sie davon wüssten. 


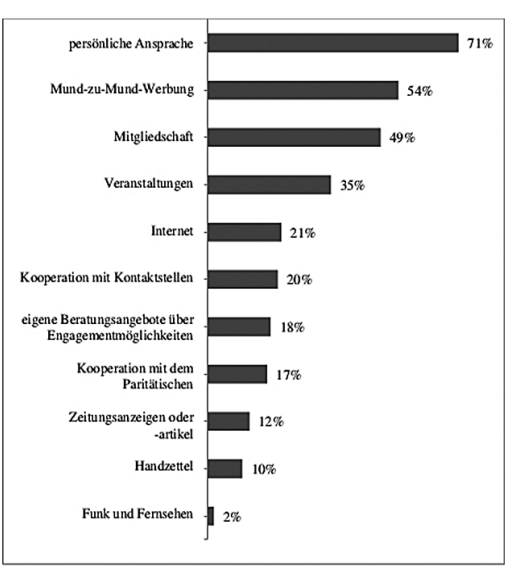

Abb. 7: Die Mehrheit der befragten sozialen Organisationen greift zur Gewinnung von Engagierten am ehesten auf Möglichkeiten der persönlichen Ansprache zurück.

$\mathrm{n}=1.586-1.631$, Skala $1-5$ (1 = »überhaupt nicht", 2 = "eher wenig", 3 = »teils/teils", 4 = "eher stark", 5 = "sehr stark «): hier Position 4 und 5 dargestellt (Backhaus-Maul/Speck/Hörnlein/ Krohn 2014)

Die meisten der befragten Organisationen verfügen neben der Möglichkeit, Engagierte aktiv in ihre Organisation einzubinden, auch über Mitglieder. Die Untersuchung erfasst, ob die Engagierten gleichzeitig auch Mitglied in der jeweiligen Organisation sind. Die Ergebnisse zeigen, dass sich dieses Verhältnis für die einzelnen Organisationen sehr unterschiedlich darstellt. In einem Drittel der Organisationen sind alle Engagierten gleichzeitig auch Mitglied der Organisation, in anderen Organisationen sind neben Mitgliedern auch Nichtmitglieder engagiert tätig. Interessant ist der Vergleich zwischen der Anzahl der engagiert tätigen Mitglieder und der Gesamtzahl aller Mitglieder in den Organisationen. Demnach binden die befragten Organisationen neben 44.946 engagiert tätigen Mitgliedern weitere 902.168 beitragszahlende Mitglieder. Hier deutet sich - bei Respekt vor Nichtengagement - innerhalb der Organisationen des Paritätischen Wohlfahrtsverbandes ein weites Feld für die Akquise von Engagierten an.

\section{Mühen der Ebene}

Bereits die wenigen im vorliegenden Beitrag präsentierten Befunde machen deutlich, dass die Freien Wohlfahrtspflege eine traditionsreiche Institution in
Deutschland ist, die einerseits wie selbstverständlich im Engagement gründet, sich andererseits gegenüber Umweltänderungen, inklusive modischer Trends, enthaltsam und zurückhaltend zeigt.

Der Umgang der Freien Wohlfahrtspflege mit Engagement und Engagierten verweist unmittelbar auf Lindbloms Theorie des "Muddling Through « - des Sich-Durchwurstelns. (6) Die Engagement-Entwicklung in der Freien Wohlfahrtspflege verläuft inkrementalistisch; Innovationen ergeben sich beim »Blick in den Rückspiegel «, um dann auf gewohntem Pfad in Sichtweite »langsam im ersten Gang vorwärts zu fahren«. Die Langsamkeit behutsamer Änderungen wirkt unter Bedingungen eines beschleunigten gesellschaftlichen Wandels schlicht unzeitgemäß und letztlich sogar problemgenierend und problemverschärfend.

Die vorliegende Untersuchung macht deutlich, dass die Bedeutung des Engagements und der Bedarf an Engagierten für die befragten Organisationen hoch sind. Dabei konstatiert ein großer Teil der Befragten Probleme bei der Gewinnung von Engagierten. Im Schatten der Sicherheit generierenden Institution scheint der akute Bedarf an Gestaltung und Entwicklung des Engagements aber nicht gesehen zu werden.

Hinzu kommt - so die qualitativen Befunde der vorliegenden Studie - dass eine heterogene und dezentrale Organisation, wie der Paritätische Wohlfahrtsverband, Engagement nicht "top down« steuern, sondern allenfalls anre-
Wohlfahrtspflege) erscheint 2014 bei Springer VS (Wiesbaden). Die Untersuchung wurde möglich durch das Engagement von Förderern, Experten und Mitarbeitenden auf Seiten des Paritätischen Wohlfahrtsverbandes - Gesamtverband und dessen Landesverbänden Berlin, Nordrhein-Westfalen und Thüringen sowie der Martin-LutherUniversität Halle-Wittenberg in Zusammenarbeit mit der Carl von Ossietzky Universität Oldenburg. Die Finanzierung erfolgte durch Förderung des Paritätischen Wohlfahrtsverbandes aus Mitteln der Aktion Mensch e. V.

(2) Diese Feststellung basiert auf den Aussagen derjenigen befragten Organisationen, die Angaben über die Zahl der Engagierten gemacht haben.

(3) Vgl. Baldas, Eugen/Bangert, Christopher (2008): Ehrenamt und freiwilliges Engagement in der Caritas. Allensbacher Repräsentativbefragung, qualitative Befragung, Ergebnisse-Perspektiven, Freiburg im Breisgau; Diakonisches Werk der Evangelischen Kirche in Deutschland e. V. (2012): Freiwilliges Engagement in Einrichtungen und Diensten der Diakonie. Eine repräsentative Studie, Stuttgart.

(4) Vgl. Baldas, Eugen/Bangert, Christopher (2008): Ehrenamt und freiwilliges Engagement in der Caritas. Allensbacher Repräsentativbefragung, qualitative Befragung, Ergebnisse-

\section{"Trotz neuer Formen: Das klassische kontinuierliche Engagement dominiert"}

gen kann. Entscheidend für die Ausprägung und Entwicklung des Engagements ist das jeweilige Selbstverständnis der Organisationen der Freien Wohlfahrtspflege, sei es als bürgergesellschaftlicher, funktionalistischer, pragmatischer oder etwa gelassen-inaktiver Organisationstyp (weiterführend Backhaus-Maul/ Speck/Hörnlein/Krohn 2014).

\section{Anmerkungen}

(1) Die Studie (vgl. Backhaus-Maul/ Speck/Hörnlein/Krohn 2014: Engagementpotenziale in der freien
Perspektiven, Freiburg; Diakonisches Werk der Evangelischen Kirche in Deutschland e. V. (2012): Freiwilliges Engagement in Einrichtungen und Diensten der Diakonie. Eine repräsentative Studie, Stuttgart.

(5) Diese Feststellung basiert auf Angaben von rund drei Vierteln derjenigen befragten Organisationen, die Angaben über die Zahl der Engagierten gemacht haben.

(6) Lindblom, Charles E. 1959: The Science of Muddling-Through, in: Public Administration Review, 19. Jg., Heft 2, S. 79-88. 